\title{
Increased genomic alteration complexity and telomere shortening in B-CLL cells resistant to radiation-induced apoptosis
}

\author{
H. Salin ${ }^{a}$ b M. Ricoul ${ }^{a} \quad$ L. Morat ${ }^{a} \quad$ L. Sabatier ${ }^{a}$ \\ a Laboratoire de Radiobiologie et d'Oncologie (LRO), CEA, DSV/iRCM, Fontenay-aux-Roses and \\ ${ }^{\mathrm{b}}$ Muséum national d'Histoire naturelle, Paris (France)
}

Accepted in revised form for publication by P. Slijepcevic, 25 September 2008.

\begin{abstract}
B-cell chronic lymphocytic leukemia (B-CLL) results in an accumulation of mature $\mathrm{CD} 5^{+} / \mathrm{CD} 23^{+} \mathrm{B}$ cells due to an uncharacterized defect in apoptotic cell death. $\mathrm{B}-\mathrm{CLL}$ is not characterized by a unique recurrent genomic alteration but rather by genomic instability giving rise frequently to several chromosomal aberrations. Besides we reported that $\sim 15 \%$ of B-CLL patients present malignant $\mathrm{B}$-cells resistant to irradiation-induced apoptosis, contrary to $\sim 85 \%$ of patients and normal human lymphocytes. Telomere length shortening is observed in radioresistant B-CLL cells. Using fluorescence in situ hybridization (FISH) and multicolour FISH, we tested whether specific chromosomal aberrations might be associated with the radioresistance of a subset of B-CLL cells and whether they are correlated with telomere shortening. In a cohort of $30 \mathrm{~B}$-CLL patients, all of
\end{abstract}

the radioresistant B-CLL cell samples exhibited homozygous or heterozygous deletion of 13q14.3 in contrast to $52 \%$ of the radiosensitive samples. In addition to the 13q14.3 deletion, ten out of the 11 radioresistant B-cell samples had another clonal genomic alteration such as trisomy 12 , deletion $17 \mathrm{p} 13.1$, mutation of the $\mathrm{p} 53$ gene or translocations in contrast to only three out of 19 radiosensitive samples. Telomere fusions and non-reciprocal translocations, hallmarks of telomere dysfunction, are not increased in radioresistant $\mathrm{B}$-CLL cells. These findings suggest (i) that the 13q14.3 deletion accompanied by another chromosomal aberration is associated with radioresistance of B-CLL cells and (ii) that telomere shortening is not causative of increased clonal chromosomal aberrations in radioresistant B-CLL cells.

Copyright $\odot 2008$ S. Karger AG, Basel
B-cell chronic lymphocytic leukemia (B-CLL), a distinct subset of human indolent lymphoma, is the most frequent leukemia of adults in the Western world. This pathology results in an accumulation of mature-looking malignant monoclonal B-cells in peripheral blood, bone marrow and lymph nodes (Guipaud et al., 2003). This accumulation is generally considered due to a defect in apoptosis rather than

\footnotetext{
Dr. Salin was supported by a post-doctoral fellowship from Commissariat à l'Energie Atomique. Work in the L.S. laboratory was supported by the ARECA project.

Request reprints from Dr. Laure Sabatier

CEA de Fontenay-aux-Roses, DSV/iRCM/LRO

18, Route du Panorama B.P.6

FR-92265 Fontenay-aux-Roses Cedex (France)

telephone: +33 1 46548351; fax: 33146548758

e-mail: laure.sabatier@cea.fr
}

to an excessive proliferation of a malignant clone, at least, at the early stage of the disease (Caligaris-Cappio, 2000). The typical phenotype of B-CLL includes expression of CD19, CD5 and CD23, but low or absent expression of CD22, FMC7, CD79b and surface immunoglobulins (IgM and $\operatorname{IgD})$. The B-CLL disease is a highly heterogeneous human malignancy, presumably reflecting specific molecular alterations in gene expression and protein activity that are thought to underlie the variable disease outcome. It is not characterized by a unique recurrent genomic alteration but rather by genomic instability frequently giving rise to several chromosomal aberrations: deletions $6 \mathrm{q} 21,11 \mathrm{q} 22 \rightarrow \mathrm{q} 23$, $13 \mathrm{q} 14,17 \mathrm{p} 13$ and trisomy 12 as the most relevant chromosomal abnormalities with unknown mechanism. Deletions in chromosomes 11 and 17 are recognized as an important pejorative prognostic factor in B-CLL, whereas patients with structural abnormalities of chromosome 13 seemed to

\begin{tabular}{lll}
\hline KARGER & $\begin{array}{l}\text { Fax }+41613061234 \\
\text { E-Mail karger@karger.ch } \\
\text { www.karger.com }\end{array}$ & $\odot 2008$ S. Karger AG, Basel \\
$1424-8581 / 08 / 1224-0343 \$ 24.50 / 0$
\end{tabular}


have a more favourable prognosis exhibiting survival probabilities similar to those with a normal karyotype (Dohner et al., 2000; Stilgenbauer et al., 2000). Short telomeres are correlated with unmutated IgVH (immunoglobulin heavy variable group) genes and are described as associated with genetic complexity and short survival (Roos et al., 2008). Moreover, the changes in the expression of genes involved in telomere maintenance suggest a global telomere dysfunction (Poncet et al., 2008). However the link of causality between telomere alterations and chromosome instability in B-CLL has not been demonstrated (Jahrsdorfer and Weiner, 2008).

Besides we have reported that the response to exogenous DNA damage may discriminate two B-CLL cell subsets with respect to subsequent triggering of apoptotic death (Guipaud et al., 2003). Thus, $\gamma$-irradiation induces in vitro apoptosis in lymphocytes derived from $\sim 85 \%$ of B-CLL patients like in normal human lymphocytes, but not in lymphocytes derived from $\sim 15 \%$ of B-CLL cases. Radioresistant B-CLL cells are characterized by alterations in DNA repair pathways and more especially by the upregulated DNA-PK dependent non-homologous end-joining (NHEJ) repair pathway (Deriano et al., 2005) that could partially contribute to the resistance to irradiation-induced apoptosis. Moreover, this DNA double-strand break (DSB) repair system seems to be more error-prone in radioresistant BCLL cells than in the radiosensitive ones (Deriano et al., 2006), which could lead to a higher accumulation of chromosomal abnormalities in radioresistant B-CLL cells (Blaise et al., 2002). These data led us to seek whether specific chromosomal aberrations might be relevant to the resistance of a subset of B-CLL cells to activate apoptotic cell death. The role of telomere dysfunction in the formation of chromosome aberrations is largely reported (reviewed in O'Hagan et al., 2002; Murnane and Sabatier, 2004). Telomere shortening leads to large chromosome instability such as end-to end fusions, gene amplification and non reciprocal translocation (Sabatier et al., 2005).

We report herein that in a cohort of $30 \mathrm{~B}$-CLL patients, all of the radioresistant B-CLL cell samples always disclosed deletion 13q14.3, associated in the large majority of the patients with another clonal genetic abnormality, contrary to the radiosensitive B-CLL cell subset. Radioresistant B-CLL cells present shorter telomeres and a higher level of clonal genomic alterations, however, those alterations are not specific to telomere shortening. We speculate that the increased genomic alterations are mainly due to error-prone DNA repair in the resistant B-CLL cell subtype.

\section{Patients, materials and methods}

Patients' selection

The patients included in the study were followed-up at PitiéSalpêtrière Hospital (Paris, France). The cell samples were selected according to their defined sensitivity to in vitro irradiation-induced apoptosis (Masdehors et al., 2000; Deriano et al., 2005). Thirty samples were selected. Nineteen out of 30 cell samples were radiosensitive versus 11 radioresistant samples.

\section{Cellular culture with mitogen stimulation}

To obtain metaphases for cytogenetic analysis, isolated peripheral blood lymphocytes were cultured at $2 \times 10^{6}$ cells $/ \mathrm{ml}$ in RPMI 1640 medium supplemented with $10 \%$ heat-inactivated fetal calf serum, L-glutamine, non-essential amino acids, $0.002 \%$ Staphylococcus aureus Cowan I (Pansorbin ${ }^{\circledR}$ Cells, Calbiochem) and $10 \mathrm{U}$ human interleukin 2 (Roche) $/ \mathrm{ml}$ for $90 \mathrm{~h}$ at $37^{\circ} \mathrm{C}$. Chromosomes were prepared by the standard protocol (Dutrillaux and Couturier, 1981). Briefly, $60 \mathrm{ng} / \mathrm{ml}$ colchicine was added for $1 \mathrm{~h} 30 \mathrm{~min}$ at $37^{\circ} \mathrm{C}$. Cells were spun at 1300 rpm for $7 \mathrm{~min}$, resuspended in $0.075 \mathrm{M} \mathrm{KCl}$, incubated for $20 \mathrm{~min}$ at $37^{\circ} \mathrm{C}$, then spun again at $1300 \mathrm{rpm}$ for $7 \mathrm{~min}$, resuspended in a fixation solution (3 vol methanol:1 vol acetic acid) and incubated overnight at $4^{\circ} \mathrm{C}$. Cells were washed once with the fixation solution before slide preparation.

\section{Telomere length detection: PNA-FISH}

We have performed telomere PNA FISH according to the protocol described previously (Sabatier et al., 2005). Briefly, slides underwent two rounds of fixation and rinses separated by a pepsin treatment. After a dehydration step, air-dried slides were hybridized with a probe mixture containing fluorescent-labeled peptide nucleic acid (PNA)(CCCTAA)3. Denaturation of the DNA was followed by hybridization at $37^{\circ} \mathrm{C}$ for $2 \mathrm{~h}$ and washes. Chromosomes were counterstained with DAPI in antifading solution.

Telomere length measurement

Telomere lengths from nine B-CLL-S (radiosensitive) cell samples and eight B-CLL-R (radioresistant) cell samples were measured with the hybridometry technique as described previously (Freulet-Marrière et al., 2004). Briefly, cell lysates were produced after $\mathrm{NaOH}$ treatment $(\mathrm{pH}>13)$ overnight and sonification of the cell pellet. Telomeric units were quantified using an enzyme hybridization assay on 96-well microtiter plates grafted with a telomeric captor sequence. A biotin-coupled tracer-oligonucleotide hybridized with telomere fragments and an enzymatic reaction was performed with a streptavidin-acetylcholinesterase conjugate, using the colorimetric method of Ellman (Freulet-Marrière et al., 2004). The OD measure was directly proportional to the number of telomeric units in the cell lysate. Mean telomere length was finally calculated from the number of telomeric units detected in a known amount of DNA issued from diploid cells.

\section{Telomerase activity}

Telomerase activities were assessed using the TRAPeze ELISA Telomerase detection kit (Oncor, Gaithersburg, MD) according to manufacturer's instructions. Assays were performed twice in independent experiments on $1 \mu \mathrm{g}$ lysates. Briefly, we incubated cell lysates in the presence of a biotinylated telomerase substrate oligonucleotide (bTS) at $30^{\circ} \mathrm{C}$ for $30 \mathrm{~min}$. Then, the extended products were amplified by PCR. After PCR, the TRAP products were tagged with biotin and DP residues. The labeled products were immobilized onto streptavidincoated microtiter plates via biotin-streptavidin interaction and then detected by anti-dinitrophenyl antibody conjugated to horseradish peroxidase substrate $\left(3,3^{\prime}, 5,5^{\prime}\right.$-tetramethylbenzidine) by measurement of the absorbance at 450 and $595 \mathrm{~nm}$. Each sample was tested with a heat inactivated $\left(85^{\circ} \mathrm{C}\right.$ for $\left.10 \mathrm{~min}\right)$ or ribonuclease treated aliquot. Reagent controls that lacked cell extracts were also systematically tested.

Detection of genomic aberrations by fluorescence in situ hybridization.

Fluorescence in situ hybridization experiments using LSI ATM/LSI p53 probe set and LSI D13S319/LSI 13q34/CEP12 probe set (Abbott, France) hybridizing respectively 11q22.3, 17p13.1, 13q14.3, 13q34 and $12 \mathrm{p} 11.1 \rightarrow$ q11 were performed according to the manufacturer's instructions. After hybridization and washing, cells were counterstained with DAPI in antifade and examined using an epifluorescence microscope equipped with a camera and appropriate filters. Images were digitized by means of the camera coupled to a digitization board, both driven by Cytovision software (Applied Imaging). Multicolor fluorescence in situ hybridization experiments were performed according to the manufacturer's instructions (Metasystem). Briefly, metaphase spreads were 

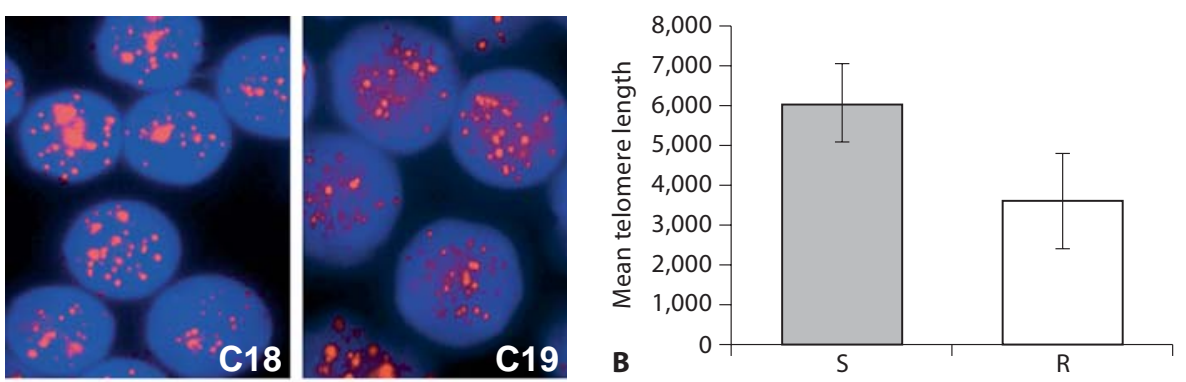

Fig. 1. Telomere shortening in radioresistant B-CLL cell samples. (A) Fluorescence in situ hybridization experiments using PNAtelomeric probe (Cy3) on nuclei from radiosensitive (C18 and $\mathrm{C} 19)$ and radioresistant (C23 and C28) cell samples. (B) Quantification of telomere length by hybridometry in nine radiosensitive (S) and eight radioresistant (R) B-CLL cells. (C) Telomerase activity measurement was performed by in vitro TRAP assay.
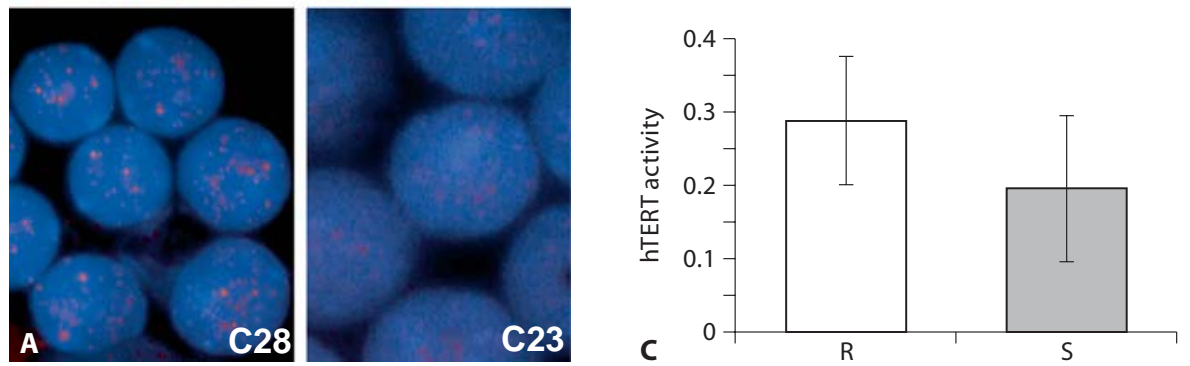

hybridized with a multicolor painting probe panel (Metasystems). After hybridization and washing, metaphase spreads were counterstained with DAPI in antifade and examined using an epifluorescence microscope equipped with a camera and appropriate filters. Images were digitized by means of the camera coupled to a digitization board, both driven by Metafer software (Metasystem). Image analysis was carried out through Isis software (Metasystem). Each chromosome was pseudocolored in a specific color, enabling an accurate identification of marker chromosomes.

\section{Results}

Shorter telomeres in all the patients with B-CLL cells resistant to irradiation-induced apoptosis

In order to compare telomere length in the two cell subtypes we performed in situ hybridization of PNA telomeric probes on interphase nuclei from ten samples (five BCLL:S, five B-CLL:R). A decrease in signal intensity is observed in B-CLL cells resistant to irradiation-induced apoptosis. This shortening is shown in Fig. 1A for the two patients from which we got samples showing an evolution from radiosensitive cells at diagnosis towards a radioresistant status several years later (cases C19/C23 and cases C18/C28).

To quantify telomere shortening we performed hybridometry techniques. This method was developed in our lab (Freulet-Marrière et al., 2004) in order to measure the number of telomeric units (TU) (TTAGGG) directly from cell lysate which presents randomly sized DNA fragments whose concentration is directly determined by an innovative $\mathrm{SYBr}$ Green based assay. This technique was applied to 17 samples (11 B-CLL:S and six B-CLL:R). Telomere length is shorter in radioresistant cells $(6.07 \mathrm{~kb}$ versus $3.58 \mathrm{~kb}$; Student $\mathrm{t}$ test: $P=0.002$ ) as shown in Fig. 1B. This telomere shortening is not correlated to a decrease in telomerase activity as demonstrated by TRAP-assay in Fig. 1C.

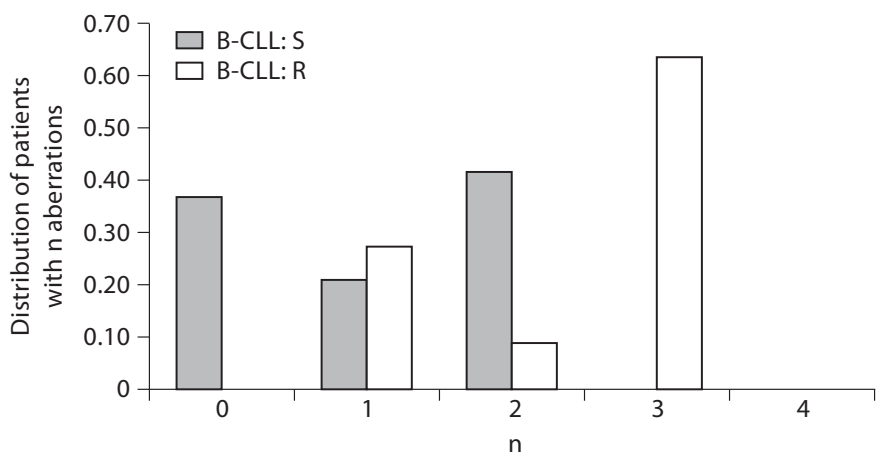

Fig. 2. Distribution of radioresistant (R) and radiosensitive (S) B-CLL samples with $0,1,2,3$ or 4 of the following genomic alterations: one deletion 13q14.3 on one chromosome 13, one deletion $13 \mathrm{q} 14.3$ on the second chromosome 13 , one deletion $17 \mathrm{p} 13.1$ and a trisomy 12 .

\section{Deletion $13 q 14.3$ in all the patients with B-CLL cells} resistant to irradiation-induced apoptosis

To investigate a correlation between the radiosensitivity status and the chromosomal aberrations of malignant Bcells, fluorescence in situ hybridization experiments using five probes hybridizing to $11 \mathrm{q} 22.3,12 \mathrm{p} 11.1 \rightarrow \mathrm{q} 11,13 \mathrm{q} 14.3$, $13 \mathrm{q} 34$ and $17 \mathrm{p} 13.1$ were performed and results analysed on metaphases and interphase nuclei for each patient. The distribution of B-CLL samples with $0,1,2,3$ or 4 genomic alterations such as one deletion 13q14.3 on one chromosome 13 , one deletion 13q14.3 on the second chromosome 13, one deletion $17 \mathrm{p} 13.1$ and a trisomy 12 revealed that the radioresistant B-CLL cells had globally more alterations than the radiosensitive samples (Fig. 2). The radiosensitive samples exhibited at most two aberrations per cell, whereas $64 \%$ of the radioresistant cases presented three alterations. More- 
Table 1. Cytogenetic data of the 30 B-CLL cases

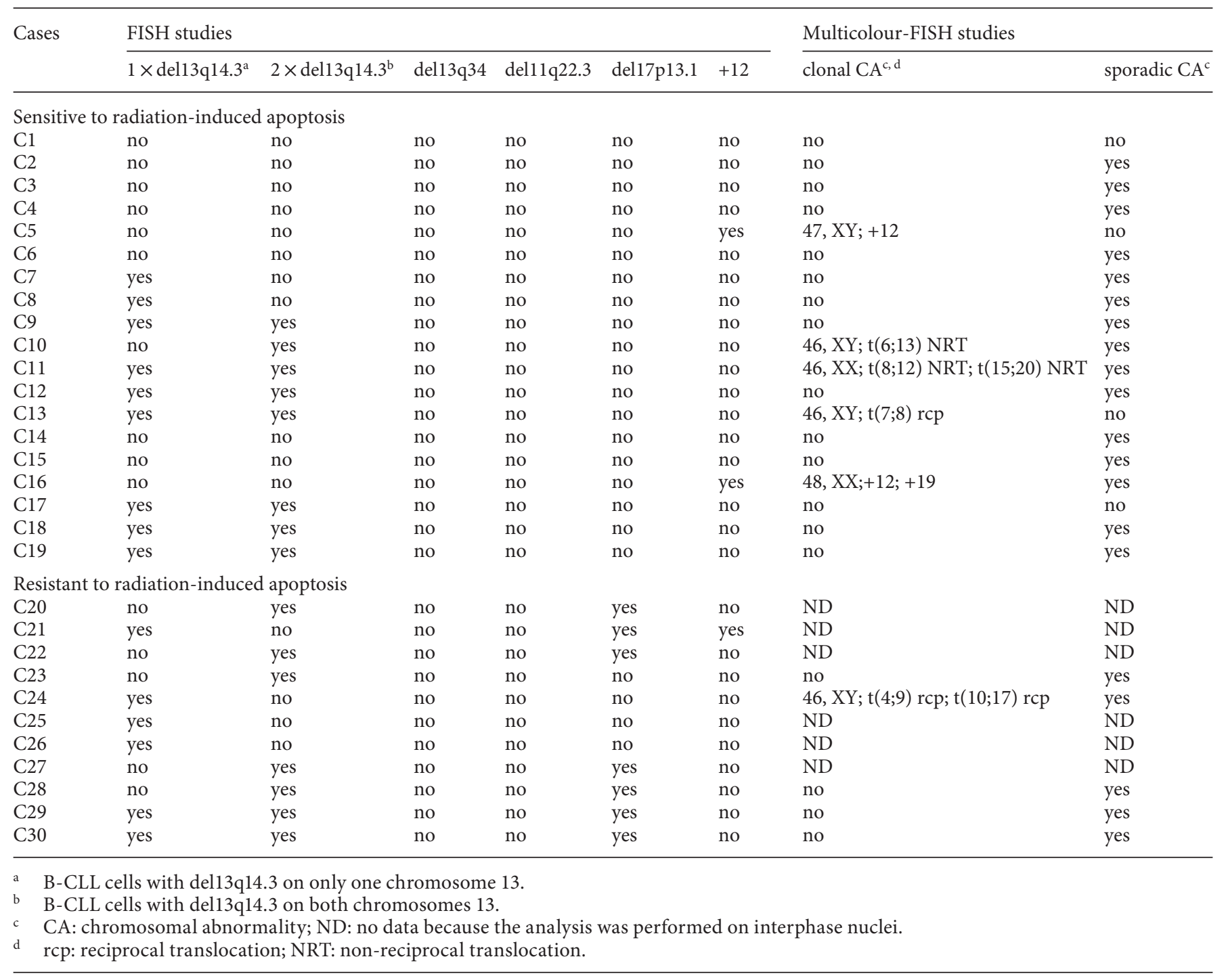

over the radioresistant cells had at least one chromosomal abnormality per cell whereas $37 \%$ of the radiosensitive samples had none of these aberrations. Among the studied chromosomal alterations, no link to the radiosensitivity status was found except for the deletion 13q14.3 (Table 1). All of the radioresistant B-CLL cell samples exhibited homozygous or heterozygous interstitial deletion at the $13 q 14.3$ region without any subtelomeric deletion 13q34 (Table 1), whereas nine out of $19 \mathrm{~B}-\mathrm{CLL}$ patients with radiosensitive $\mathrm{B}$-cells did not (Table 1). This difference between the radiosensitive and radioresistant groups is significant (Student t test: $P=0.000645)$.

To supplement the analysis of chromosomal aberrations, multicolor fluorescence in situ hybridization experiments (M-FISH) were carried out. The results of analyzed genetic abnormalities for the studied patients are recapitulated in Table 1. A global analysis reveals that the mean number of clonal genetic alterations per patient was significantly higher in the radioresistant subset than in the radiosensitive subset (respectively, 2.81 and 1.31; Student t test: $P=0.00032$ ) and that the average number of chromosomes per metaphase involved in sporadic chromosomal aberrations was 0.42 for the sensitive group and 1.07 for the resistant group (data not shown). More precisely, in addition to the deletion $13 q 14.3$, ten out of 11 radioresistant cell samples presented another clonal genomic alteration such as trisomy 12, deletion 17p13.1, or some clonal translocations, whereas seven out of the ten radiosensitive samples with deletion 13q14.3 did not exhibit any additional clonal chromosomal abnormality. This difference is significant (Student t test: $P=$ $0.00025)$. It is worth noting that the three other radiosensitive samples with deletion $13 \mathrm{q} 14.3$ presented translocations as additional clonal chromosomal aberrations but did not have any trisomy 12 and any deletion $17 \mathrm{p} 13.1$ contrary to 
nine out of 11 radioresistant samples. This difference between the radiosensitive and radioresistant groups is also significant (Student t test: $P=0.000275$ ). The added clonal aberrations are not characterized by the occurrence of endto-end fusions, none was detected in the $30 \mathrm{~B}$-CLL samples. Clonal Non Reciprocal Translocations (NRT) were observed in cells from two B-CLL:S and none in B-CLL:R (Table 1). Sporadic NRT are detected in both radiosensitive and radioresistant cell subtypes (6/19 and 2/5 respectively); no sporadic end-to-end fusions were detected in the radiosensitive subset and a single fusion in the radioresistant cell subtypes (data not shown).

Interestingly, for the two patients included in the list of patients with radioresistant cells (Cases C23 and C28 in Table 1) that presented previously radiosensitive malignant $\mathrm{B}$ cells (respectively, C19 and C18), homozygous and heterozygous deletions at 13q14.3 had been observed as the only clonal chromosomal aberrations in the malignant cells sensitive to irradiation-induced apoptosis for these two patients. For one of these, the change from radiosensitive to radioresistant status came with the appearance of the deletion 17p13.1 (C28). The analysis of sporadic rearrangements showed seven NRT in 25 metaphases (C18) versus nine NRT and one fusion in 28 metaphases (C28). No sporadic NRT is observed in C19 and only one in C23.

\section{Discussion}

Using hybridometry, FISH and M-FISH techniques, we investigated a correlation between the radiosensitivity status of B-CLL cells, telomere length and their genomic alterations. In contrast to the radiosensitive B-CLL cell samples in which the deletion 13q14.3 was the only detectable cytogenetic abnormality, the deletion 13q14.3 was accompanied by additional clonal cytogenetic alterations in all radioresistant cases except one. Shorter telomeres are detected in radioresistant B-CLL cell samples. Hallmarks of telomere induced chromosome rearrangements such as end-to-end fusions and non reciprocal translocations are not found to be increased in radioresistant B-cell samples.

Deletion $13 q 14.3$ in $100 \%$ of the radioresistant B-CLL cell cases versus $52 \%$ of the radiosensitive subset

The patients with radioresistant B-CLL cells represent about $15 \%$ of B-CLL patients (Guipaud et al., 2003) and 45 to $62 \%$ B-CLL patients exhibit a deletion 13q14.3 (Dohner et al., 2000; Stilgenbauer et al., 2002; Sindelarova et al., 2005). Our results revealed a deletion 13q14.3 in all of the radioresistant B-CLL cell samples, which means that 30 to $47 \%$ of B-CLL patients have both the deletion and radiosensitive cells. Therefore 35 to $55 \%$ of the radiosensitive subset should exhibit a deletion at the $13 \mathrm{q} 14.3$ region, which is consistent with our results: $52 \%$ of radiosensitive cell samples analyzed by FISH experiments showed a deletion 13q14.3.
Is the deletion 13q14.3 added to another clonal genomic alteration associated with the radioresistant status of

$B$-CLL cells?

The association of the homozygous or heterozygous deletion 13q14.3 with trisomy 12 , deletion $17 \mathrm{p} 13.1$ or TP53 mutation is a characteristic found in nine out of the $11 \mathrm{ra}$ dioresistant B-CLL cell samples and in none of the radiosensitive samples. Moreover for one of two patients whose radiosensitivity status changed, the deletion 13q14.3 was already observed when the patient had radiosensitive B-CLL cells but the deletion $17 \mathrm{p} 11.3$ was seen only when the B-CLL cells became resistant. All of these results lead us to suggest that the radioresistant status of B-CLL cells is associated with deletion 13q14.3 accompanied by trisomy 12, deletion $17 \mathrm{p} 13.1$ or TP53 mutation. However one radioresistant sample which had no trisomy 12 and no deletion 17p13.1 (TP53 mutational status not done) presented a clonal translocation as an additional genomic alteration. So the radioresistant status appears to be linked to multiple aberrations including always the 13q14.3 deletion, irrespective of the additional clonal genomic abnormalities. As some radiosensitive BCLL cases also had the 13q14.3 deletion plus another clonal translocation, the question is whether the 13q14.3 deletion accompanied by another chromosomal abnormality could predict the change from the radiosensitive to the radioresistant status. To answer this question, the radiosensitivity evolution of patients with multiple aberrations including deletion 13q14.3 and with, at the present, radiosensitive Bcells, would have to be followed up in further experiments and compared with the evolution of patients with radiosensitive cells and the 13q14.3 deletion as a single aberration.

\section{Deletion 13q14.3 and additional chromosomal}

aberration: a prognosis value or not?

Dohner's data showed in 2000 that the patients with a 13 q14 deletion as a single aberration had the longest estimated median treatment-free interval and survival time as compared to the groups with deletion $17 \mathrm{p}$, deletion $11 \mathrm{q}$ or trisomy 12 , whereas patients with multiple abnormalities had a worse outcome than patients with a single aberration (Dohner et al., 1997a, b, 2000; Stilgenbauer et al., 2000). Consistently, the patients with radioresistant B-CLL cells belong to the group of patients with complex chromosomal aberrations and clinical resistance to treatment appeared to be linked to the resistance of B-CLL cells to in vitro irradiation-induced apoptosis (Vallat et al., 2003). Moreover, the evolution towards the radioresistant status for one patient was accompanied by (i) the addition of deletion $17 \mathrm{p} 11.3$ to the 13q14.3 deletion and (ii) a clinical aggravation of the pathology requiring immediate treatment. Thus, if the association of the 13q14.3 deletion with another clonal chromosomal abnormality can predict the change from the radiosensitive to radioresistant status, then the detection of several chromosomal abnormalities including the 13q14.3 deletion in the B-CLL cells might constitute a prognosis factor of the pathology progression to a more aggressive form. This is supported by some B-CLL cases presented by Rosenwald et al. (2004). In their study, the mutational $\operatorname{IgV}_{\mathrm{H}}$ 
status, considered as the most revealing prognostic factor, was determined for nine B-CLL patients. The mutated $\operatorname{IgV}_{\mathrm{H}}$ status is a good prognostic indicator contrary to the unmutated $\operatorname{IgV}_{\mathrm{H}}$ status. Consistently with our findings, the BCLL cases with unmutated $\operatorname{IgV}_{\mathrm{H}}$ and deletion 13q14 had additional chromosomal abnormalities, whereas the patients with mutated $\mathrm{IgV}_{\mathrm{H}}$ and deletion $13 \mathrm{q} 14$ did not. However, Sindelarova et al.'s study (2005) did not prove association of any chromosomal abnormality with a mutated or unmutated $\mathrm{IgV}_{\mathrm{H}}$ clone. It is worth noting that the association of the deletion 13q14.3 with another chromosomal abnormality, required to be linked to a bad prognosis, constitutes a characteristic of B-CLL since in other malignancies such as multiple myeloma (Avet-Loiseau et al., 2000, 2002), the deletion $13 \mathrm{q} 14$ as a single aberration is sufficient to be associated with a short survival time.

\section{Putative origin of genomic alteration pattern in the} radioresistant $B$-CLL subset.

The mechanisms leading to the chromosomal alterations classically associated with B-CLL are unknown. As increasing numbers of alterations are observed in B-CLL with short telomeres, as telomeres are twice as short in radioresistant B-CLL cell samples and as short telomeres induce genomic alterations, it is tempting to speculate that telomeres may play a role in the origin of chromosomal alterations. We observed that radiosensitive status in the absence of aberrations or in the presence of del13q14 as the only cytogenetic abnormality were associated with long telomeres. In contrast, the radioresistant status and del13q14 with or without additional clonal alterations were associated with short telomeres. Thus shorter telomeres are associated with increased chromosome alterations that confirm the results published by Roos et al. (2008). However we failed to detect an increase of telomere-induced instability in radioresistant subsets such as non reciprocal translocations or end-to-end fusions. Although telomeres shorten, the mean length of telomeres reaches $3 \mathrm{~kb}$ in the radioresistant B-CLL cells. Such a length could keep the large majority of telomeric ends above the 'destabilization threshold'. Moreover the low but detectable telomerase activity could maintain a 'protected' telomeric status and could restrain telomere-driven instability. Thus telomere driven chromosome alteration would not be the major mechanism involved in the accumulation of genomic alterations and would not be detectable in the relatively small (30) group of patients studied. However alterations of telomere maintenance could have preceded telomere shortening and could be involved in the occurrence of NRT in both radiosensitive and radioresistant B-CLL groups.

An attractive hypothesis that could explain the origin of genomic alterations accumulated in radioresistant B-CLL cells would involve the DNA repair process. We have shown that DNA-PK dependent NHEJ repair pathway is upregulated and more error-prone in radioresistant B-CLL cells compared to radiosensitive B-CLL cells (Deriano et al., $2005,2006)$. The upregulation of this DNA-DSB repair system could partially explain (i) the resistance to DSB-induced apoptosis such as induced by radiation and (ii) the more aggressive progression of the pathology associated with the radioresistant B-CLL group, since a 'hyper-NHEJ' activity in B-CLL cells would lead to resistance to genotoxic treatments and to more severe disease (Muller et al., 1998).

Our findings have shown that the deletion $13 \mathrm{q} 14.3 \mathrm{ac}-$ companied by another chromosomal aberration is associated with the radioresistance of B-CLL cells. Telomere shortening would not be causative of increased clonal chromosomal aberrations in radioresistant B-CLL cells whereas the upregulation of an error-prone DNA repair process could lead to the accumulation of alteration in the radioresistant B-CLL group. It will be interesting to discover the consequences of global telomere dysfunction (Poncet et al., 2008) in B-CLL and their interconnections with DNA repair.

\section{Acknowledgements}

The authors are grateful to all the volunteer leukemic blood donors at Service d'Hématologie Biologique, Hôpital Pitié-Salpêtrière, Paris (Pr H. Merle-Béral). We thank Gabrielle Potocki de Montalk, Florence Nguyen-Khac, Sylvie Baudet and Géraldine Pottier for their assistance and Irène Baccelli and Jozo Delic for helpful discussion.

\section{References}

Avet-Loiseau H, Daviet A, Sauner S, Bataille R, In- Blaise R, Alapetite C, Masdehors P, Merle-Beral H, tergroupe Francophone du Myelome: Chromosome 13 abnormalities in multiple myeloma are mostly monosomy 13. Br J Haematol 111:11161117 (2000)

Avet-Loiseau H, Facon T, Grosbois B, Magrangeas F, Rapp MJ, et al, Intergroupe Francophone du Myelome: Oncogenesis of multiple myeloma: $14 \mathrm{q} 32$ and $13 \mathrm{q}$ chromosomal abnormalities are not randomly distributed, but correlate with natural history, immunological features, and clinical presentation. Blood 99:2185-2191 (2002). Roulin C, et al: High levels of chromosome aberrations correlate with impaired in vitro radiation-induced apoptosis and DNA repair in human B-chronic lymphocytic leukaemia cells Int J Radiat Biol 78:671-679 (2002).

Caligaris-Cappio F: Biology of chronic lymphocytic leukemia. Rev Clin Exp Hematol 4:5-21 (2000).

Delic J, Morange M, Magdelenat H: Ubiquitin pathway involvement in human lymphocyte $\gamma$-irradiation-induced apoptosis. Mol Cell Biol 13: 4875-4883 (1993).

-Deriano L, Guipaud O, Merle-Beral H, Binet JL, Ricoul M, et al: Human chronic lymphocytic leukemia B cells can escape DNA damage-induced apoptosis through the non-homologous end-joining DNA repair pathway. Blood 105: 4776-4783 (2005).
Deriano L, Merle-Béral H, Guipaud O, Sabatier L, Delic J: Mutagenicity of non-homologous end joining DNA repair in a resistant subset of human chronic lymphocytic leukemia B cells. Br J Haematol 133:520-525 (2006).

Dohner H, Stilgenbauer S, James MR, Benner A, Weilguni T, et al: 11q deletions identify a new subset of B-cell chronic lymphocytic leukemia characterized by extensive nodal involvement and inferior prognosis. Blood 89:2516-2522 (1997a).

Dohner H, Stilgenbauer S, Fischer K, Bentz M, Lichter P: Cytogenetic and molecular cytogenetic analysis of $B$ cell chronic lymphocytic leukemia: specific chromosome aberrations identify prognostic subgroups of patients and point to loci of candidate genes. Leukemia 11 Suppl 2 . S19-24 (1997b). 
Dohner H, Stilgenbauer S, Benner A, Leupolt E, Krober A, et al: Genomic aberrations and survival in chronic lymphocytic leukemia. $\mathrm{N}$ Engl J Med 343:1910-1916 (2000).

Dutrillaux B, Couturier J: La pratique de l'analyse chromosomique. 1st ed, p 86 (Masson, Paris 1981).

Freulet-Marriere MA, Potocki-Veronese G, Deverre JR, Sabatier L: Rapid method for mean telomere length measurement directly from cell lysates. Biochem Biophys Res Commun 314: 950-956 (2004).

- Guipaud O, Deriano L, Salin H, Vallat L, Sabatier L, et al: B-cell chronic lymphocytic leukaemia: a polymorphic family unified by genomic features. Lancet Oncol 4:505-514 (2003).

Jahrsdörfer B, Weiner GJ: Short telomeres in BCLL: the chicken or the egg? Blood 111:5756 (2008).

-Masdehors P, Merle-Béral H, Maloum K, Ömura S, Magdelenat H, Delic J: Deregulation of the ubiquitin system and p53 proteolysis modify the apoptotic response in B-CLL lymphocytes. Blood 96:269-274 (2000).
Muller C, Christodoulopoulos G, Salles B, Panasci L: DNA-Dependent protein kinase activity correlates with clinical and in vitro sensitivity of chronic lymphocytic leukemia lymphocytes to nitrogen mustards. Blood 92:2213-2219 (1998).

Murnane JP, Sabatier L: Chromosome rearrangements resulting from telomere dysfunction and their role in cancer. Bioessays 26:1164-1174 (2004).

O’Hagan RC, Chang S, Maser RS, Mohan R, Artandi SE, et al: Telomere dysfunction provokes regional amplification and deletion in cancer genomes. Cancer Cell 2:149-155 (2002).

Poncet D, Belleville A, t'kint de Roodenbeke C, Roborel de Climens A, Ben Simon E, et al: Changes in the expression of telomere maintenance genes suggest global telomere dysfunction in Bchronic lymphocytic leukemia. Blood 111: 2388-2391 (2008).

Roos G, Kröber A, Grabowski P, Kienle D, Bühler A, et al: Short telomeres are associated with genetic complexity, high-risk genomic aberrations, and short survival in chronic lymphocytic leukemia. Blood 111:2246-2252 (2008).

Rosenwald A, Chuang EY, Davis RE, Wiestner A, Alizadeh AA, et al: Fludarabine treatment of patients with chronic lymphocy tic leukemia induces a p53-dependent gene expression response. Blood 104:1428-1434 (2004).
Sabatier L, Ricoul M, Pottier G, Murnane JP: The loss of a single telomere can result in instability of multiple chromosomes in a human tumor cell line. Mol Cancer Res 3:139-150 (2005).

Sindelarova L, Michalova K, Zemanova Z, Ransdorfova S, Brezinova J, et al: Incidence of chromosomal anomalies detected with FISH and their clinical correlations in B-chronic lymphocytic leukemia. Cancer Genet Cytogenet 160:27-34 (2005).

Stilgenbauer S, Lichter P, Dohner H: Genetic features of B-cell chronic lymphocytic leukemia. Rev Clin Exp Hematol 4:48-72 (2000).

Stilgenbauer S, Bullinger L, Lichter P, Dohner H: Genetics of chronic lymphocytic leukemia: genomic aberrations and $\mathrm{V}(\mathrm{H})$ gene mutation status in pathogenesis and clinical course. Leukemia 16:993-1007 (2002).

Vallat L, Magdelenat H, Merle-Beral H, Masdehors P, Potocki de Montalk G, et al: The resistance of B-CLL cells to DNA damage-induced apoptosis defined by DNA microarrays. Blood 101:45984606 (2003). 